[2] Choy EH, Panayi GS. Cytokine pathways and joint inflammation in rheumatoid arthritis. The New England journal of medicine. 2001;344(12):90716

[3] Mclnnes IB, Schett G. The pathogenesis of rheumatoid arthritis. The New England journal of medicine. 2011;365(23):2205-19.

[4] Connor AM, Mahomed N, Gandhi R, Keystone EC, Berger SA. TNFalpha modulates protein degradation pathways in rheumatoid arthritis synovial fibroblasts. Arthritis research \& therapy. 2012;14(2):R62.

[5] Rinaldi N, Schwarz-Eywill M, Weis D, Leppelmann-Jansen P, Lukoschek $M$, Keilholz $U$, et al. Increased expression of integrins on fibroblast-like synoviocytes from rheumatoid arthritis in vitro correlates with enhanced binding to extracellular matrix proteins. Annals of the rheumatic diseases. 1997;56(1):45-51.

[6] Bueno M, Lai YC, Romero Y, Brands J, St Croix CM, Kamga C, et al. PINK1 deficiency impairs mitochondrial homeostasis and promotes lung fibrosis. The Journal of clinical investigation. 2015;125(2):521-38.

[7] Ding WX, Yin XM. Mitophagy: mechanisms, pathophysiological roles, and analysis. Biological chemistry. 2012;393(7):547-64.

Acknowledgement: This research was supported by the Basic Research Program through the National Research Foundation of Korea (NRF) funded by the MSIP (2014R1A1A1004857)

Disclosure of Interests: None declared

DOI: 10.1136/annrheumdis-2019-eular.4641

\section{SAT0051 BARICITINIB IMPROVES JOINT MOBILITY AFTER INJURY IN A RODENT FORCED-AMBULATION MODEL}

Kelly Knopp, Akihiko Kato, Theron Wall, Jeff S. Mcdermott, Eric S. Nisenbaum, Benjamin Adams, Michael Johnson. Eli Lilly and Company, Indianapolis, United States of America

Background: Movement-evoked pain and impaired joint mobility are common comorbidities in inflammatory diseases such as Rheumatoid Arthritis (RA) and Osteoarthritis. The Janus kinase (JAK) pathway has been implicated in both inflammation and chronic pain. Clinical data suggests that baricitinib, a selective JAK $1 / 2$ inhibitor, can robustly and rapidly alleviate pain in RA. ${ }^{1}$ Utilizing a rodent forced-ambulation model, we have previously shown attenuation of gait deficits with analgesics such as opioids, nonsteroidal anti-inflammatory drugs (NSAIDs), and an anti-NGF (Nerve Growth Factor) antibody. ${ }^{2}$ However, in this same model a fusion protein blocker of TNF $\alpha$ (tumor necrosis factor alpha) signaling failed to demonstrate efficacy though inflammation was decreased. The present work investigated the potential of baricitinib, recently approved for treatment of $\mathrm{RA}$, to reduce inflammagen-induced joint pain and gait impairment in this model of joint inflammation mediated pain.

Objectives: To determine if JAK $1 / 2$ pathway inhibition is effective in treating inflammagen-induced joint pain and gait impairment.

Methods: Unilateral joint injury was induced in female Sprague Dawley rats (Harlan, Indianapolis, IN, USA) by unilateral intra-articular injection of $20 \mu \mathrm{g}$ Complete Freund's Adjuvant (CFA). Using the GaitScan (CleverSys Inc., Reston, VA) treadmill system, a composite gait score comprising of range of motion, normalized stance distance, stance/swing ratio, and paw print size was evaluated over 3 days post-injection. ${ }^{2}$ Rats were treated with vehicle, positive control (40 mg/kg Tramadol), or clinically relevant (based on plasma levels) increasing doses of baricitinib $(1,3$, or $10 \mathrm{mg} /$ kg p.o., 2-hrs prior to each test, q.d.). Dorsal root ganglion (DRG) were harvested post-gait evaluation and Total STAT3 (Cell Signaling, \#4904) and phospho-STAT3 (Y705) (Cell Signaling, \#9131) protein levels were examined via immunoblotting. The $p$-values were derived from repeated measures ANOVAs

Results: In rat DRG homogenates, baricitinib significantly decreased phospho-STAT3 (Y705) protein levels in a dose-dependent manner $(p<0.01)$ with a significant effect after a $3 \mathrm{mg} / \mathrm{kg}$ dose and a maximal response after a $10 \mathrm{mg} / \mathrm{kg}$ in both the ipsilateral (right) and contralateral (left) sides. Total STAT3 protein levels remained unchanged. Similarly, treatment with baricitinib significantly improved composite gait score at the 10 $\mathrm{mg} / \mathrm{kg}$ dose $(p<0.05)$ by Day 3 .

Conclusion: These data indicate that treatment with baricitinib attenuates CFA-induced joint deficits, a surrogate measure of joint pain. This effect correlated with the pharmacodynamic inhibition of JAK-STAT signaling in DRGs. These data support a role for JAK-STAT signaling in pain signaling and provide an opportunity to investigate the potential mechanism of action of baricitinib in joint pain.



Figure 1. Representative still images from video recordings of animals during gait analysis at baseline (Top Panel), 3 Days post-CFA and vehicle treatment (Middle Panel) and 3 Days post-CFA with $10 \mathrm{mg} / \mathrm{kg}$ daily baricitinib treatment (Bottom panel). The ventral image of the rat illustrates the same part of the gait cycle (ipsilateral stance phase initiation) across treatment groups to highlight impairment in the CFA-injected limb (Red Box) vs Baselin and apparent improvement following $10 \mathrm{mg} / \mathrm{kg}$ baricitinib treatment. The panels on the left provide examples of software-detected paw prints for each treatment group which show that CFA + vehicle treatment produced a small, closed-toed paw print (Middle) which appears restored to near baseline levels following baricitinib treatment (Bottom).

\section{REFERENCES}

[1] Keystone. Ann Rheum Dis. 2017;76(11):1853-1861.

[2] Adams. Osteoarthritis Cartilage. 2016;24(11):1928-1939.

Disclosure of Interests: Kelly Knopp Shareholder of: Eli Lilly and Company, Employee of: Eli Lilly and Company, Akihiko Kato Shareholder of: Eli Lilly and Company, Employee of: Eli Lilly and Company, Theron Wall Shareholder of: Eli Lilly and Company, Employee of: Eli Lilly and Company, Jeff S Mcdermott Shareholder of: Eli Lilly and Company, Employee of: Eli Lilly and Company, Eric S Nisenbaum Shareholder of: Eli Lilly and Company, Employee of: Eli Lilly and Company, Benjamin Adams Shareholder of: Eli Lilly and Company, Employee of: Eli Lilly and Company, Michael Johnson Shareholder of: Eli Lilly and Company, Employee of: Eli Lilly and Company

DOI: 10.1136/annrheumdis-2019-eular.1751

\section{SAT0052 PHOTODYNAMIC THERAPY TARGETING ACTIVATED FIBROBLASTS INDUCES SYNOVIAL CELL DEATH IN EXPERIMENTAL ARTHRITIS}

Daphne Dorst ${ }^{1}$, Mark Rijpkema ${ }^{1}$, Mijke Buitinga ${ }^{2}$, Peter Laverman ${ }^{1}$, Marti Boss ${ }^{1}$, Christian $\mathrm{Klein}^{3}$, Anne Freimoser-Grundschober ${ }^{3}$, Birgitte Walgreen ${ }^{1}$, Peter van der Kraan ${ }^{1}$, Martin Gotthardt ${ }^{1}$, Marije Koenders ${ }^{4} .{ }^{1}$ Radboudumc, Nijmegen, Netherlands; ${ }^{2}$ KULeuven, Leuven, Belgium; ${ }^{3}$ Roche Pharmaceutical Research and Early Development, Innovation Center Zurich, Sclieren, Switzerland;

${ }^{1}$ Radboudumc, Nijmegen, Netherlands

Background: Activated synovial fibroblasts (SF) contribute to rheumatoid arthritis (RA) by producing a multitude of cytokines, chemokines and proteases thus aggravating disease. Activated SF can be distinguished from quiescent fibroblasts by their expression of fibroblast activation protein (FAP). Selective depletion of FAP+ SF in inflamed joints could decrease their contribution to the arthritis process and thus constitute a viable treatment option. Further focussing of the treatment to only those areas affected by the disease can be accomplished by applying targeted photodynamic therapy (tPDT). In tPDT a light sensitive molecule, a photosensitizer (PS), is conjugated to a targeting moiety. Upon activation by light this construct produces reactive oxygen species, killing the targeted cells. Objectives: To this end we developed and tested a therapy that selectively depletes activated SF by targeting FAP on these cells with an 
antibody, $28 \mathrm{H} 1$, to which the PS, IRDye700DX, for tPDT is attached. Here we investigated the feasibility of using FAP-tPDT to induce cell death in murine arthritic synovium ex vivo.

Methods: After conjugation of the IDRye700DX to $28 \mathrm{H} 1$ (28H1-700DX), binding and specificity of the conjugate was determined. Subsequently, tPDT efficiency in vitro was established using a 3 T3 fibroblast cell line stably transfected with FAP. Biodistribution using an $\left[{ }^{111} \mathrm{In}\right] \mathrm{In}-\mathrm{DTPA}-28 \mathrm{H} 1$ conjugate with and without IRDye700DX was performed in healthy C57BL/6N mice as well as in C57BL/6N mice with antigen induced arthritis (AIA). Finally, the potential of FAP-tPDT to induce targeted cell death in the synovial lining was determined by treating knee joints from mice with AIA ex vivo.

Results: Conjugation of IRDye700DX to the antibody did not negatively influence the immunoreactive fraction or binding capacity of the conjugate (94.7\% for $28 \mathrm{H} 1-700 \mathrm{DX})$. $28 \mathrm{H} 1-700 \mathrm{DX}$ was able to efficiently induce FAP-specific cell death in vitro. At $17.6 \mathrm{~J} / \mathrm{cm}^{2}$ radiant exposure, $89.24 \%$ $\pm 3.67 \%$ of fibroblasts died in the group incubated with antibody compared to control incubated with buffer only $(p<0.001)$. Biodistribution of the compound with the PS showed increased accumulation in the liver compared to the antibody without PS $(31.46 \pm 5.49 \%$ injected dose per gram tissue $(\% \mathrm{ID} / \mathrm{g})$ versus $5.32 \pm 1.17 \% \mathrm{ID} / \mathrm{g}$ for the antibody with or without PS, respectively $(\mathrm{p}<0.001))$. However, despite this increased clearance to the liver, accumulation in the inflamed joints was increased in the group injected with the antibody-PS construct $(1.61 \pm 0.08 \%$ ID versus $1.13 \pm 0.06 \% \mathrm{ID}$ for the antibody with or without PS $(p<0.001))$. Interestingly, ex vivo FAP-tPDT of knee joints of arthritic mice caused significant photo-bleaching of the PS (19.69 $\pm 2.02 \%$ fluorescent signal remaining versus $96.00 \pm 25.98 \%$ compared to the unexposed control at baseline, $\mathrm{p}=0.047$ ). Furthermore FAP-tPDT induced marked apoptosis as was indicated by an increased staining of the markers caspase- 3 and yH2AX evident in the synovium of treated knee joints.

Conclusion: Here we demonstrated the feasibility of conjugating a PS to an antibody targeting FAP on activated SF without negatively impacting the binding capacity thereof. Furthermore we showed that this construct can then be used to deliver cell specific cytotoxicity through TPDT both in vitro and ex vivo in a mouse model of arthritis. This approach may have therapeutic potential in the treatment of RA.

Disclosure of Interests: Daphne Dorst: None declared, Mark Rijpkema: None declared, Mijke Buitinga: None declared, Peter Laverman: None declared, Marti Boss: None declared, Christian Klein Employee of: Roche pharmaceutics, Anne Freimoser-Grundschober Employee of: Roche pharmaceutics, Birgitte Walgreen: None declared, Peter van der Kraan: None declared, Martin Gotthardt: None declared, Marije Koenders: None declared

DOI: 10.1136/annrheumdis-2019-eular.7252

\section{SAT0053 \\ GLYCOSYLATION IN MAMMALS PROTECTS CITRULLINATED CHEMOKINES FROM PARTIAL DEGRADATION}

Olexandr Korchynskyi ${ }^{1,2,3} .{ }^{1}$ Rzeszow University, Medical Faculty, Rzeszow Poland; ${ }^{2}$ Institute of Cell Biology, Natl.Acad.Scie. of Ukraine, Lviv, Ukraine; ${ }^{3}$ Academic Medical Centre, University of Amsterdam, Amsterdam, Netherlands

Background: Citrullination is a postranslational modification of specific proteins by peptidylarginine deiminase (PAD) activities. Nowadays citrullination is recognized as a hallmark of rheutamoid arthritis and other autoimmune diseases. In our recent study we have shown a presence of citrullinated chemokines epithelial-derived monocyte chemotactic protein-1 (MCP-1/ CCL2), macrophage inflammatory protein-1 $\alpha$ (MIP-1 $\alpha / C C L 3)$ and neutrophil-activating peptide 78 (ENA-78/CXCL5) in biological fluids collected from patients suffering from rheumatoid arthritis (RA) versus non-autoimmune arthritis diseases and demonstrated citrullinated ENA-78/CXCL5 as an efficient macrophage chemoattractant in a contrast with non-citrullinated ENA-78/CXCL5. In our current work we found that citrullinated in vitro bacterially produced chemokines cannot be efficiently used as the standards in modified enzyme linked immunosorbent assay (ELISA) assays designed to detect citrullinated chemokines.

Objectives: In our work we aimed generate a procedure for preparation of stable citrullinated chemokines suitable for research aplications and to validate a hypothesis that posttranslational modifications occurring in mammalian cells that can stabilize chemokines can also protect citrullinated chemokines from quick degradation.

Methods: MCP-1/CCL2 and MIP-1 $\alpha /$ CCL3 were cloned from total RNA isolated from synovial fibroblasts obtained from RA patient. Both bacterially-produced and mammalian cells-produced recombinant human chemokines were citrullinated by commercial rabbit PAD2. Success in citrullination was confirmed with Western blotting and mass-spectrometry. Citrullinated chemokine concentrations were measured by modified sandwich ELISA assays.

Results: Both commercially available and self-made bacterially produced chemokines MCP-1/CCL2, MIP-1 $\alpha /$ CCL3 and ENA-78/CXCL5 undergo quick partial degradation upon their in vitro citrullination by PAD2 and cannot be detected with either Western blotting or mass-spectrometry. At the same time mammalian cells-produced properly glycosylated MCP-1/ CCL2, MIP-1 $\alpha /$ CCL3 and ENA-78/CXCL5 can be efficiently citrullinated and successfully used as the standards in modified ELISA assays as well as in bioassays.

Conclusion: Glycosylation that is lucking in bacterially-produced proteins but occurs in mammalian cells stabilizes citrullinated chemokines thus protecting them from quickly ongoing partial degradation.

BioEthics Committee Approval: All human subject samples were collected after approval by the Institutional Review Board of the Academic Medical Center/University of Amsterdam, Amsterdam, The Netherlands (Protocol MEC 07/079 \#10.17.0708) and provision of informed consent by the patients.

\section{REFERENCE}

[1] Yoshida K., Korchynskyi O., Tak P.-P., Isozaki T., Ruth J.H., Campbell P L., Baeten D.L., Gerlag D.M., Amin M.A., and. Koch A.E. (2014) Arthritis and Rheumat 66, No 10, P.2716-2727.

Disclosure of Interests: None declared

DOI: 10.1136/annrheumdis-2019-eular.8072

\section{SAT0054 INVESTIGATING MECHANISMS OF AUTOANTIBODY INDUCED PAIN, BONE LOSS AND ARTHRITIS DEVELOPMENT}

Akilan Krishnamurthy ${ }^{1}$, Yogan Kisten ${ }^{1}$, Katalin Sandor $^{2}$, Alexandra Circiumaru ${ }^{1}$, Gustaf Wigerblad $^{1}$, Peter Damberg ${ }^{3}$, Koji Sakurabas ${ }^{1}$, Heidi Wähämaa ${ }^{3}$, Patrik Jarvolli ${ }^{4}$, Juan Jimenez Jimenez Andrade ${ }^{5}$, Vivianne Malmström ${ }^{1}$, Lars Klareskog ${ }^{1}$, Camilla Svensson ${ }^{2}$, Bence Réthi ${ }^{1}$, Anca Catrina ${ }^{1} .{ }^{1}$ Karolinska Institutet, Rheumatology unit, Medicine, stockholm, Sweden; ${ }^{2}$ Karolinska Institutet, Physiology and Pharmacology, stockholm, Sweden; ${ }^{3}$ Karolinska Institutet, Karolinska Experimental Research and Imaging Centre (KERIC), stockholm, Sweden; ${ }^{4}$ Karolinska Institutet, stockholm, Sweden; ${ }^{5}$ Universidad Autónoma de Tamaulipas, Reynosa, Tamaulipas, Mexico

Background: In rheumatoid arthritis (RA), autoantibodies against citrullinated proteins (ACPAs) have been reported to be associated with bone loss, pain and tenosynovitis prior to disease onset.

Objectives: We aimed to investigate if transfer of human ACPAs into mice could reproduce these clinical observations.

Methods: Monoclonal ACPA (1325:04C03 and 1325:01B09) and contro (1362:01E02) antibodies (mAbs) were generated from synovial plasma or memory B cells of RA patients. 2mg of combination of monoclonal ACPAs or control antibody were injected in BALB/c female mice (12-16 Weeks) with or without a consecutive intra-articular injection of LPS after 8 days. Pain-like behavior was monitored by measuring mechanical hypersensitivity using von Frey filaments every 3 days and estimation by up-down Dixon method. Bone mineral density was measured by microCT. Using specially designed mobilization casts, dedicated mouse MR coils, and gadolinium enhanced contrast medium, the hind limbs of these mice were scanned and evaluated for any signs of soft tissue joint inflammation. Blinded to ACPA and controls, the MRI images were scored for the presence of synovial thickening, effusion and tendon inflammatory changes by 3 readers in consensus.

Results: ACPAs (1325:04C03 and 1325:01B09) induced significantly more pronounced pain-like behavior (lasting for at least 4 weeks) and reduction of the trabecular bone thickness in the hind limbs, whereas no such effect was seen with the control mabs generated in the same way as the monoclonal ACPAs. While no macroscopic sign of joint inflammation could be detected, preliminary MRI data shows that sub-clinical joint inflammation (such as tenosynovitis) in mice injected with ACPAs but not those injected with control mAb. Intra-articular LPS injection resulted in significantly increased prolonged mechanical hypersensitivity in mice initially receiving sub-optimal doses of monoclonal ACPA as compared to those receiving control mAb. This was associated with higher levels of sub-clinical inflammation (as shown by MRI scans) in ACPA injected mice.

Conclusion: We show that ACPA induces pain-like behavior, bone loss and sub-clinical inflammation in mice, a model that mimics the pre-clinical state of ACPA positive RA. 